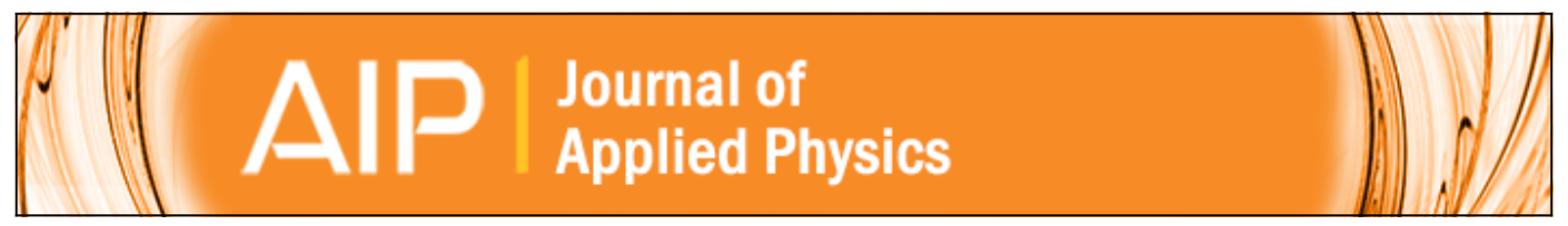

Homoepitaxial diamond growth for the control of surface conductive carrier transport properties

Oliver A Williams and Richard B Jackman

Citation: Journal of Applied Physics 96, 3742 (2004); doi: 10.1063/1.1789275

View online: http://dx.doi.org/10.1063/1.1789275

View Table of Contents: http://scitation.aip.org/content/aip/journal/jap/96/7?ver=pdfcov

Published by the AIP Publishing 


\title{
Homoepitaxial diamond growth for the control of surface conductive carrier transport properties
}

\author{
Oliver A Williams ${ }^{\text {a) }}$ and Richard B Jackman \\ Department of Electronic and Electrical Engineering, University College London, Torrington Place, \\ London WC1E 7JE, United Kingdom
}

(Received 20 January 2004; accepted 11 July 2004)

\begin{abstract}
Growth of high quality diamond for surface conductive device applications is demonstrated. Mobility values higher than $140 \mathrm{~cm}^{2} \mathrm{~V}^{-1} \mathrm{~s}^{-1}$ at sheet carrier concentrations of $2.5 \times 10^{12} \mathrm{~cm}^{-2}$ were achieved using a high growth rate process. Furthermore, control over the carrier transport statistics is demonstrated on both single crystal and polycrystalline diamond. This process allows the production of high quality electronic grade diamond with ability to tune carrier transport statistics. The mechanism behind this process is discussed. (C) 2004 American Institute of Physics.
\end{abstract}

[DOI: $10.1063 / 1.1789275]$

\section{INTRODUCTION}

Since its discovery in the late 1980s, hydrogen surface conductivity has been the source of much investigation in the field of diamond electronics. ${ }^{1}$ Early Hall measurements confirmed its nature as $p$-type ${ }^{2}$ and subsequent investigations measured an extremely low activation energy. ${ }^{3,4}$ High performance devices have been fabricated utilizing this surface conductive layer, ${ }^{5-9}$ however its origin is still under debate. ${ }^{10-13}$ As it is not a dopant in the conventional sense, control over the carrier transport values to date has been elusive. Moderate success has been achieved by moderate annealing treatments in air to partially oxidize the surface, but this also increases the sheet resistivity which is obviously undesirable for device applications. ${ }^{14}$ The work in this paper uses the diamond growth process as a way of controlling these carrier transport statistics. This method produces high quality material at a low cost with control over device characteristics.

\section{EXPERIMENTAL METHODS}

All films were grown in an Astex PDS-18 plasma deposition system, with a $2.45 \mathrm{GHz} 5 \mathrm{~kW}$ microwave source and a cooled stage. Temperature measurements were made using a Williamson Pro 92 two-color pyrometer. The growth conditions were $800{ }^{\circ} \mathrm{C}, 120$ torr, $2.5-3 \mathrm{~kW}$, with a gas phase of 478 SCCM hydrogen, 20 SCCM methane, and 2 SCCM oxygen (SCCM denotes cubic centimeter per minute at STP). The temperature was controlled by the incident microwave power. This process has a growth rate around $8-10 \mu \mathrm{m} / \mathrm{h}$, as published elsewhere. ${ }^{15}$ The diamond substrates were Sumitomo/Element 6 type $\mathrm{Ib}\{100\} /\{111\}$ oriented HPHT single crystal and Diamonex HFCVD randomly oriented polycrystalline black diamond. Various sizes were grown 4-100 $\mathrm{mm}^{2}$ area. All of these substrates contained substantial quantities of nitrogen and hence it was not possible to generate the surface conductive layer with a hydrogen

\footnotetext{
${ }^{a)}$ Author to whom correspondence should be addressed; electronic mail: oliverwilliams@mac.com
}

plasma alone. This effect has been reported elsewhere and a thin layer of high purity diamond is required to produce surface conductivity. ${ }^{16}$ Prior to deposition, all substrates were acid-cleaned in $200{ }^{\circ} \mathrm{C}$ sulfuric acid and ammonia persufhate solution to remove graphite and metallic contaminants from the HPHT process. The substrates were ramped to the final growth microwave power and temperature in a hydrogen plasma to clean the surface and reduce the misalignment angle. ${ }^{17}$ Once the system was stable, methane gas was added and the microwave power varied to settle the temperature at $800{ }^{\circ} \mathrm{C}$. After the deposition duration, the samples were cooled in a hydrogen plasma. This process removes any residual non-sp ${ }_{3}$ carbon from the surface as a result of the interrupted high growth rate process and generates $p$-type conductivity. However, it should be noted that by simply cooling in hydrogen ambient after deposition, $p$-type conductivity is still observed, but occasionally small graphitic deposits are observed on the surface by AFM, which are detrimental to device fabrication. Au contacts were thermally evaporated in the van der Pauw configuration at a base pressure lower than $2 \times 10^{-7}$ mbar. Hall measurements were made using a modified Lakeshore Cryotronics 7504 Hall measurement system, with an advanced research systems helium atmosphere based cryostat. Scanning tunnel microscopy (STM) measurements were made in air using a nanosurf easyscan STM, with the surface conductive layer of the films shorted to ground using silver paint.

\section{RESULTS}

Figure 1 demonstrates the high quality surface obtainable by this growth regime. This film is only $1 \mu \mathrm{m}$ thick, and the surface roughness is less than $1 \mathrm{~nm}$ rms. The STM image is also extremely uniform over this area. The sheet conductivity values recorded for this sample in Fig. 2 show a decrease in conductivity with decreasing temperature, with an activation energy of $18 \mathrm{meV}$. This particular sample yields a straight line and hence a single activation energy; however, this was not always the case with other samples. Therefore, the single activation energy recorded here should be observed with some caution. The Hall statistics for this sample 


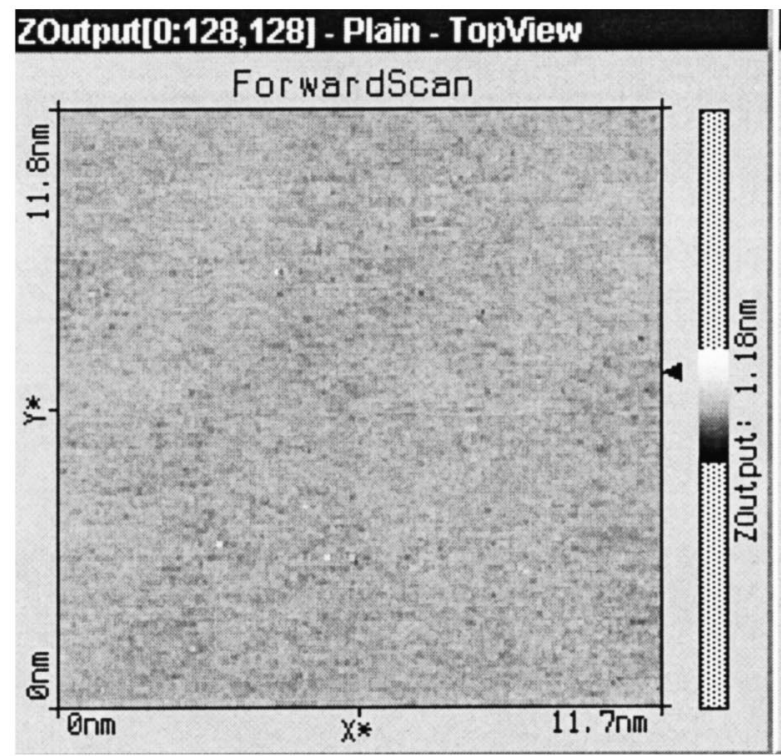

ZOutput[0:128,128] - Raw - LineView

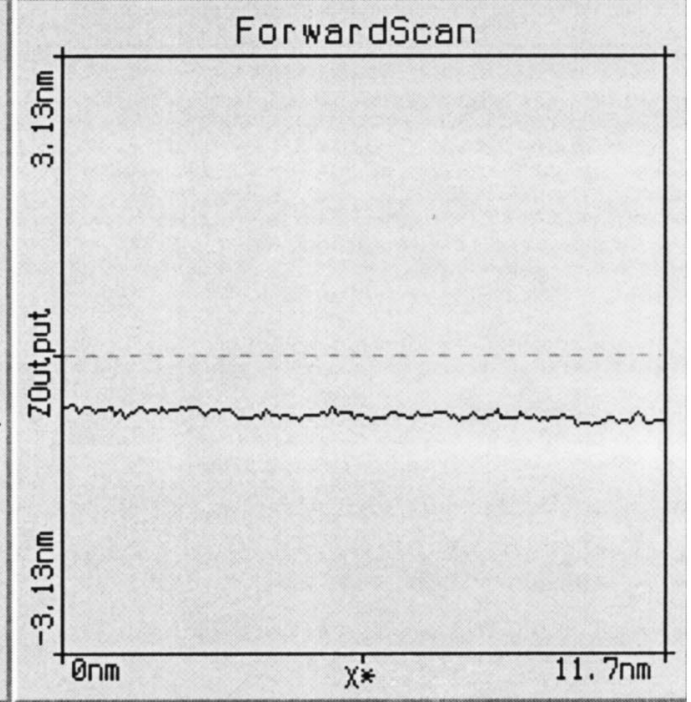

FIG. 1. STM of $1 \mu \mathrm{m}$ thick diamond film grown at $8 \mu \mathrm{m} / \mathrm{h}$.

are shown as a function of temperature in Fig. 3. Here the left axis and the filled circles show the sheet carrier concentration increasing over an order of magnitude from 2.5 $\times 10^{12}$ to $6 \times 10^{13} \mathrm{~cm}^{-2}$ over this temperature range. At lower temperature it was impossible to obtain a Hall signal due to the size and instability of the Hall offset voltage. The problems of Hall offset voltage instability in diamond are detailed elsewhere. ${ }^{18}$ The right-hand axis and hollow squares of Fig. 3 show the decrease in mobility over two orders of magnitude from greater than $140 \mathrm{~cm}^{2} \mathrm{~V}^{-1} \mathrm{~s}^{-1}$ to less than $1 \mathrm{~cm}^{2} \mathrm{~V}^{-1} \mathrm{~s}^{-1}$ with decreasing temperature. This low value of mobility also complicates obtaining Hall measurements at lower temperatures, coupled with the aforementioned high resistivity leading to increased Hall offset voltage. Figure 4 shows mobility values against sheet carrier concentrations for various samples grown by this technique. It can be seen from this plot that the values correlate, i.e., higher sheet carrier concentrations yield lower mobility values. These values range from a mobility $140 \mathrm{~cm}^{2} \mathrm{~V}^{-1} \mathrm{~s}^{-1}$ at the rate of 2.5 $\times 10^{12} \mathrm{~cm}^{-2}$ to a substantially lower mobility at

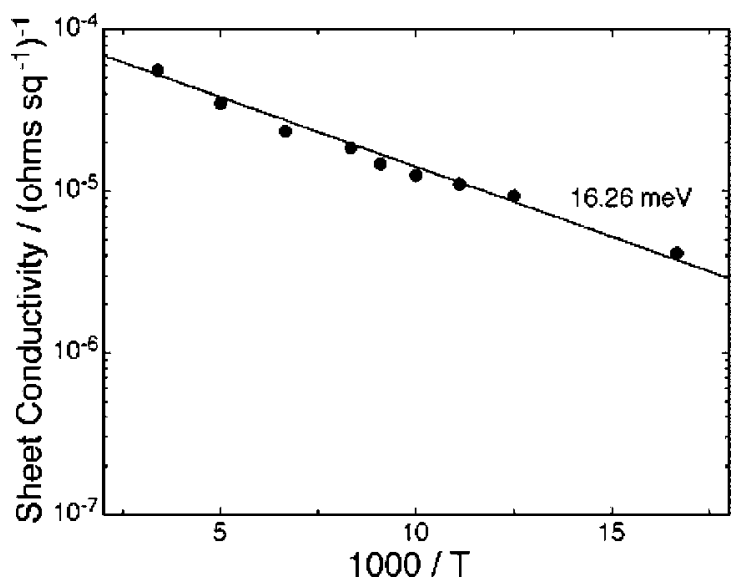

FIG. 2. Sheet resistivity against temperature for $1 \mu \mathrm{m}$ thick film on HPHT type $1 \mathrm{~b} 100$ diamond.
$22 \mathrm{~cm}^{2} \mathrm{~V}^{-1} \mathrm{~s}^{-1}$ at the rate of $8 \times 10^{13} \mathrm{~cm}^{-2}$. The process by which this variation in mobility/sheet carrier concentration is achieved is shown in Fig. 5. It can be seen from this figure that the sheet carrier concentration values increase with the thickness of the grown epitaxial layer. There is some significant scatter on this trend due to various other interacting variables, but the overall trend of sheet carrier concentrations increasing from $2.5 \times 10^{12} \mathrm{~cm}^{-2}$ with a $1 \mu \mathrm{m}$ thick overlayer to $8 \times 10^{13} \mathrm{~cm}^{-2}$ for an $8 \mu \mathrm{m}$ thick overlayer is clearly visible. Figure 6 shows the STM image of one of the $8 \mu \mathrm{m}$ thick layers. It is clear from this image that the film is significantly rougher, with the presence of some particulates on the surface, which could be unepitaxial crystallites.

A similar approach to the above was tried on polycrystalline black HFCVD material. This material has the advantage of much lower cost and the possibility of large area

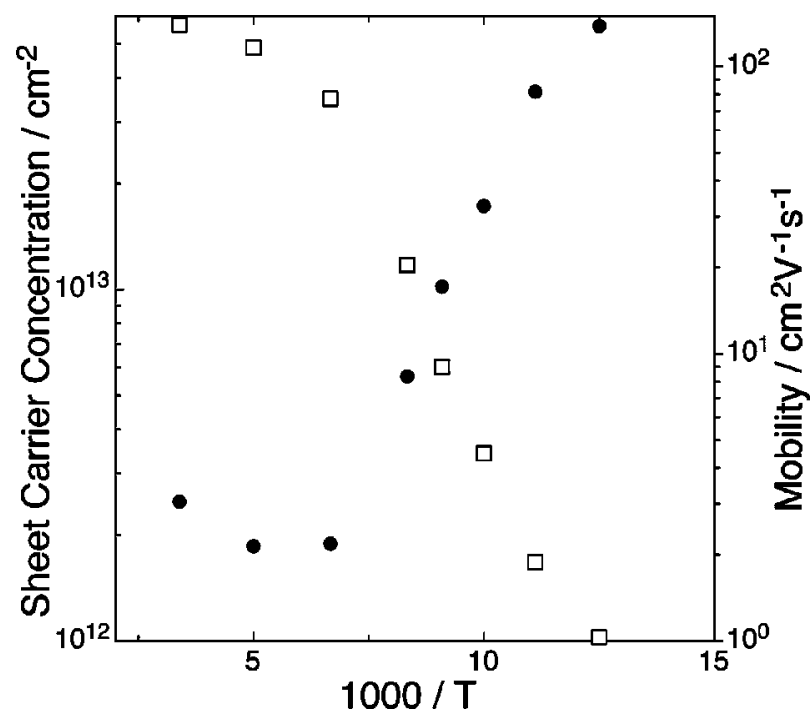

FIG. 3. Hall effect statistics of $1 \mu \mathrm{m}$ thick film on HPHT type $1 \mathrm{~b} 100$ diamond; (○) black circles $=$ mobility, $(\square)$ clear squares $=$ sheet carrier concentration. 


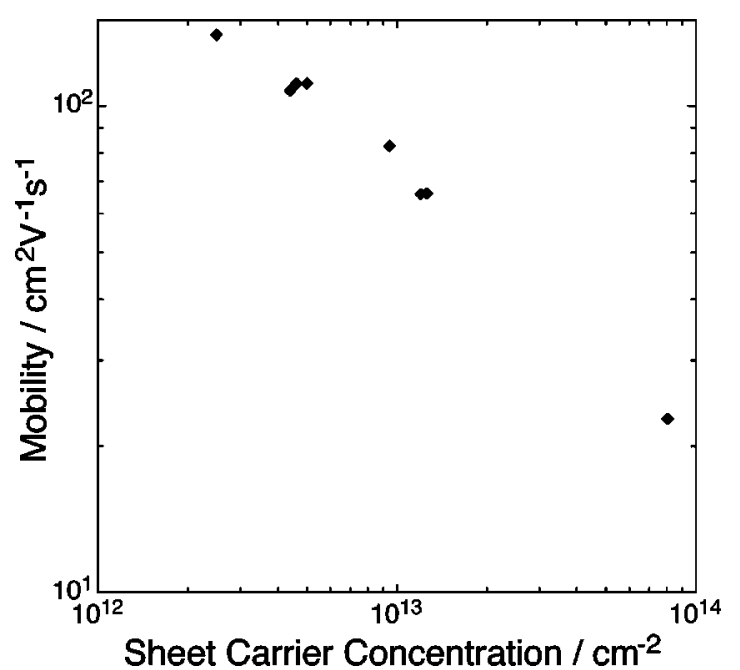

FIG. 4. Mobility against carrier concentration for homoepitaxial diamond grown on HPHT type $1 \mathrm{~b}$ substrates.

deposition. A $1 \mu \mathrm{m}$ thick film was grown on substrates of thickness $100-1000 \mu \mathrm{m}$, and a STM image of the $300 \mu \mathrm{m}$ substrate is shown in Fig. 7. It can be seen from this figure that even though the structure of the film is fundamentally polycrystalline, the image is bright and unifrom within the grain and somewhat darker at the grain boundaries. The sheet resistivities of these films are ploted in Fig. 8. It can be seen that the thicker films give a lower resistivity, droping from $8 \times 10^{5}$ to $5 \times 10^{3} \Omega /$ sq. It can be seen from Fig. 9 that this decrease in resistivity is due to an associated increase in sheet carrier concentration from $2 \times 10^{12}$ to $8 \times 10^{13} \mathrm{~cm}^{-2}$.

The Hall data of all the films grown by this method are illustrated in Fig. 10. This figure shows mobility values against sheet carrier concentration. While the figure can be seen as confusing, closer inspection with regards to the legend shows that the values of Sumitomo $\{100\}$ substrates correlate as mentioned in Fig. 5. Added to this data is the date for Element $6\{100\}$ substrates, which broadly follow

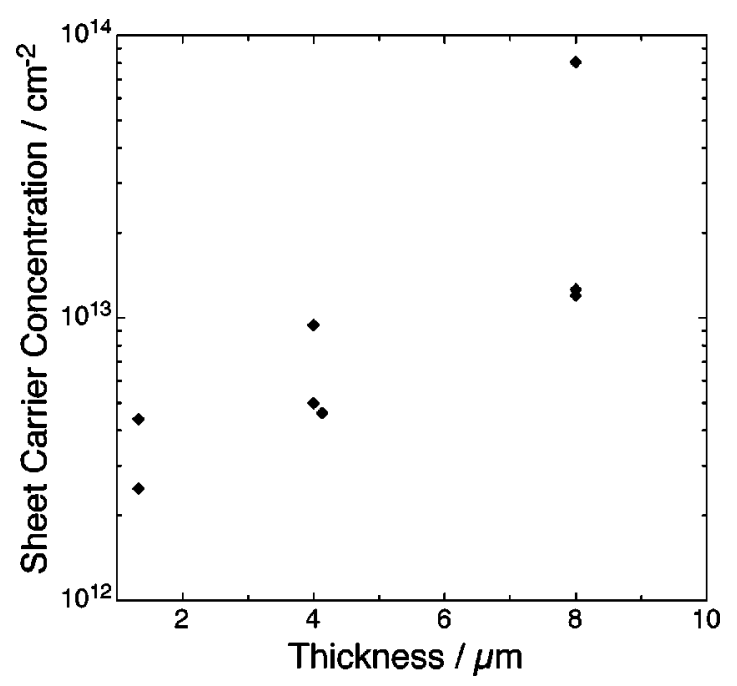

FIG. 5. Carrier concentration against thickness for homoepitaxial diamond grown on HPHT type $1 \mathrm{~b}$ substrates.

the same trend. The polycrystalline material can be seen to be confined the fundamently lower mobility values and more scattered than the single crystal data. Sumitomo $\{111\}$ material can be seen to be confined to higher sheet carrier concentrations and lower mobilities.

\section{DISCUSSION}

This approach is clearly viable for the production of high quality material for surface conductive device applications. The extreme smoothness shown in Fig. 1 is highly desirable for advanced lithography. It should also be noted that for a STM image to be uniform, both the conductivity and surface must be uniform. This means that the conductivity due to hydrogen surface conductivity is also uniform. Further information on the conductivity of this film is given in Fig. 2. The decrease in conductivity with decreasing temperature is as expected. The very low activation energy has been reported

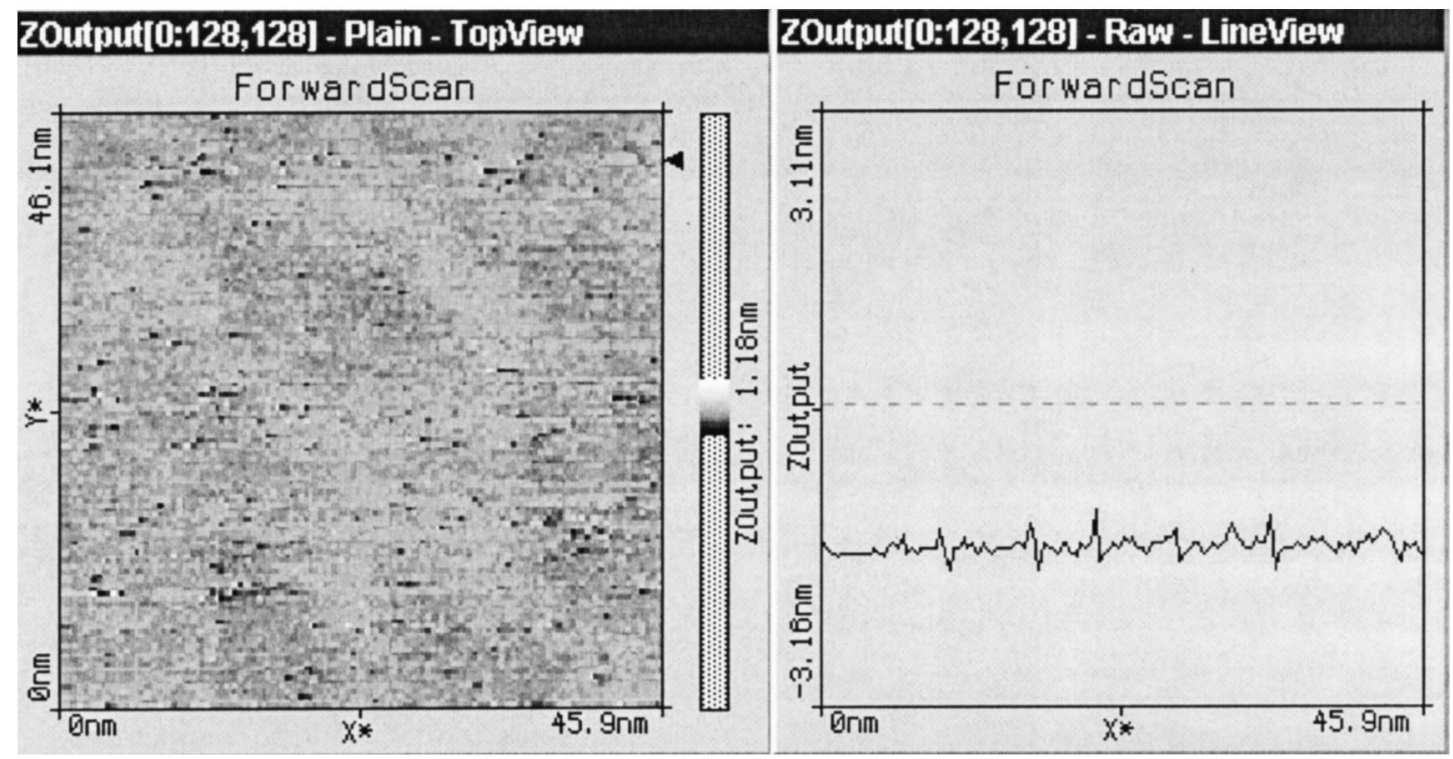

FIG. 6. STM image of $8 \mu \mathrm{m}$ thick film on HPHT type $1 \mathrm{~b} 100$ diamond. 


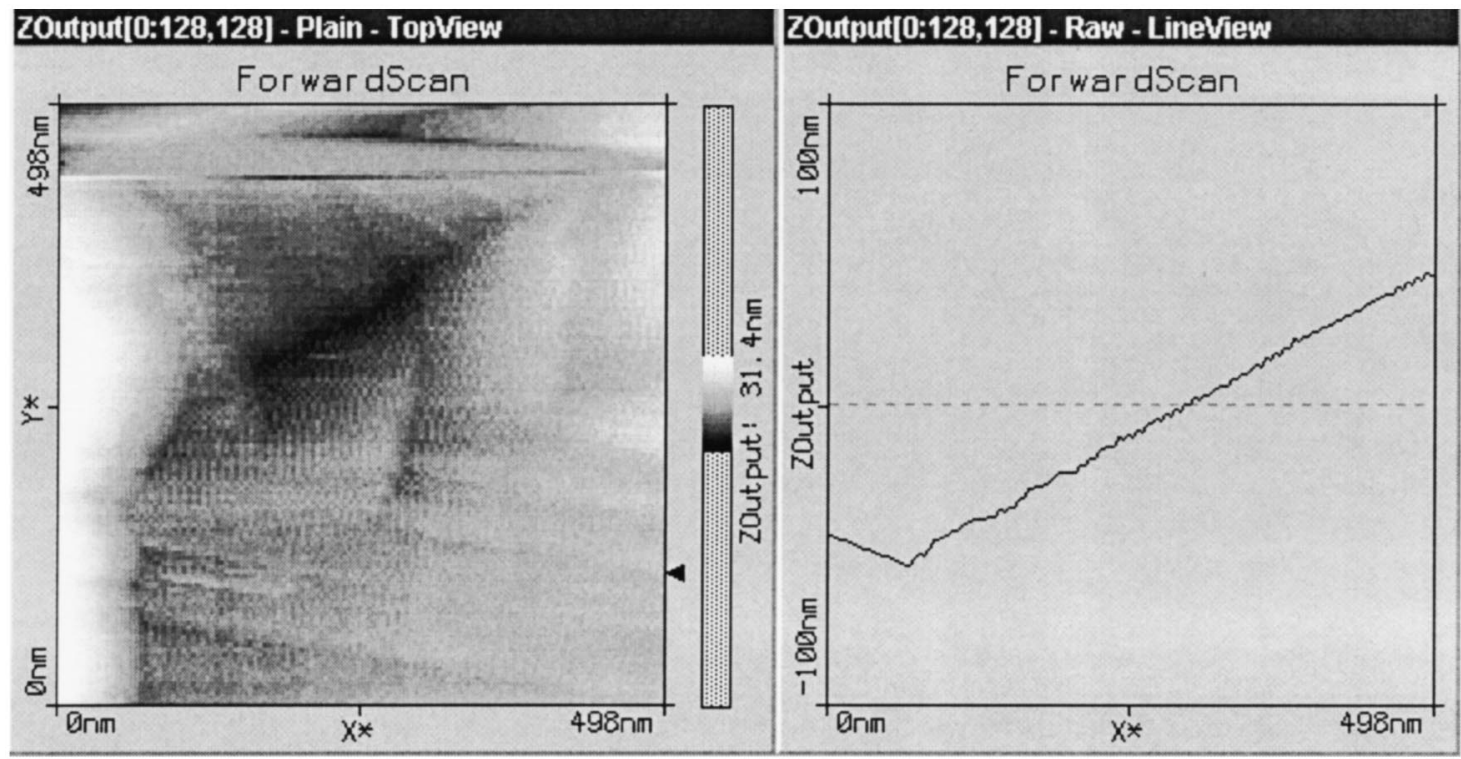

FIG. 7. STM image of $1 \mu \mathrm{m}$ thick film on HFCVD polycrystalline diamond substrate.

elsewhere and has also been shown to be environmentally dependent; ${ }^{19}$ thus it should not be seen as an evidence of conventional doping. In fact, a single activation energy is rarely observed in the case of surface conductive diamond. ${ }^{4,20}$ The room temperature value is among the highest recorded for surface conductive diamond. It can be seen from Fig. 3 that the sheet carrier concentration actually increases with decreasing temperature, which is in contradiction with the conventional device theory. The reason for this has been documented in detail elsewhere. ${ }^{13,18}$ Briefly, the results obtained here can be explained by considering the dipole formed by the differing electronegativities of the carbon and hydrogen atoms at the hydrogen terminated surface. ${ }^{5}$ This dipole can be considered to be responsible for both the origin of hydrogen surface conductivity and the mechanism of the carrier transport that is observed. It is thought that holes are created in the near-surface region by electron out diffusion into adsorbates in a water, or similar, layer at the diamond surface. ${ }^{10,21,22}$ These holes are also confined to the

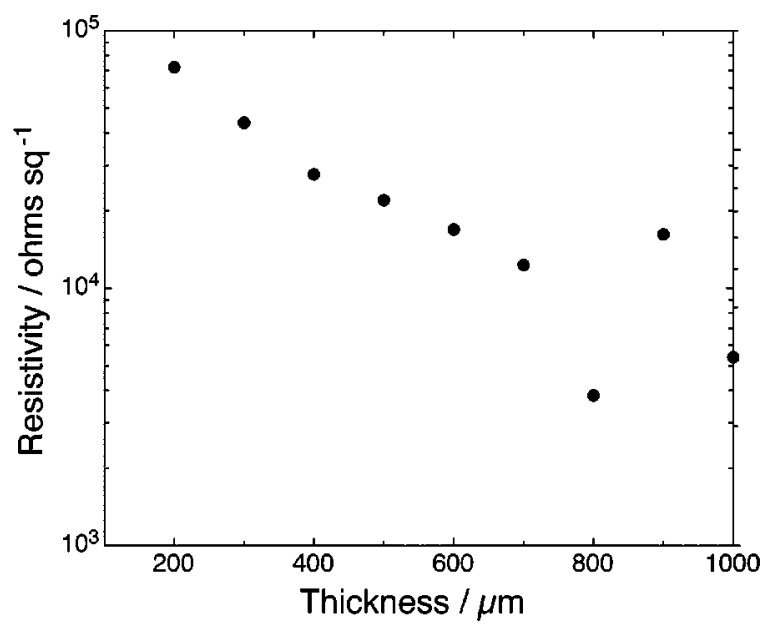

FIG. 8. Sheet resistivity against substrate thickness for $1 \mu \mathrm{m}$ thick films grown on HFCVD polycrystalline black diamond. near surface by the negative charge at the carbon atom in the dipole. As the diamond is cooled, the holes have less thermal energy and hence become increasingly confined at the surface by the dipole. This results in an increase in sheet carrier concentration. However, this confinement increases carrier scattering both by surface roughness and Coulomb interaction, resulting in a reduction in mobility with decreasing temperature. This decrease in mobility (see Fig. 3) dominates and thus the sheet conductivity decreases on cooling. The correlation between mobility values and sheet carrier concentrations for diamond grown on Sumitomo $\{100\}$ substrates is shown in Fig. 4. It can be seen from this figure that higher sheet carrier concentrations result in lower mobility values. However, the origin of this effect cannot be due to increased ionized impurity scattering as surface conductivity is not a conventional form of doping. It is possible that if the increased sheet carrier concentration is due to increasing confinement of the surface dipole, then the mobility will be reduced by this Coulomb interaction and the scattering due

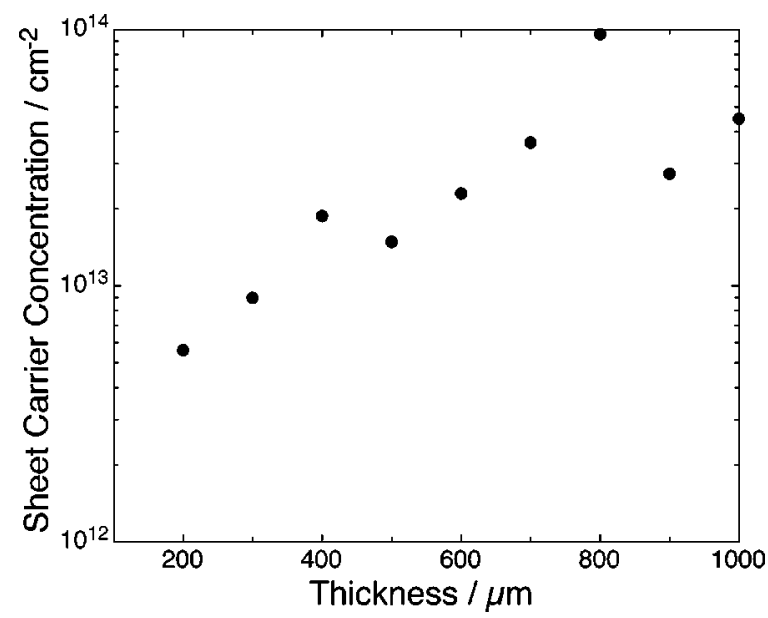

FIG. 9. Sheet carrier concentration against substrate thickness for $1 \mu \mathrm{m}$ thick films grown on HFCVD polycrystalline black diamond 


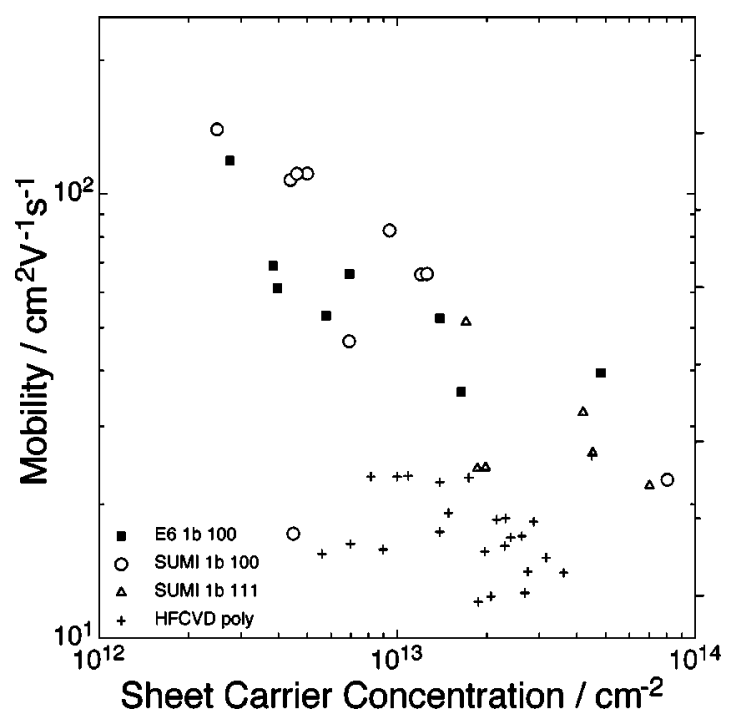

FIG. 10. Mobility against sheet carrier concentration for all grown films.

to the roughness of the surface. However, there are other mechanisms that must be considered when viewing these data. If the data are plotted as sheet carrier concentration against layer thickness as in Fig. 5, one can see a general trend towards higher carrier concentrations at greater layer thicknesses. In general, as is not expected in surface conductivity, the carriers are confined to a maximum of the first 20 $\mathrm{nm}$ from the surface. ${ }^{5,11,14}$ One possible cause is that the holes due to the hydrogen termination are compensated by deep traps due to the high concentration of nitrogen in the type Ib substrate. This would explain why thicker layers have a higher carrier concentration, because the layer would be growing away from this region. However, this explanation is not sufficient, as it has been empirically shown by others that only a very thin layer $(\sim 15 \mathrm{~nm})$ is necessary to achieve high sheet carrier densities $\left(\sim 1 \times 10^{13} \mathrm{~cm}^{-2}\right) .{ }^{16}$ What was not reported in this work though was the surface roughness of the resulting film. Figure 6 shows the roughness of an $8 \mu \mathrm{m}$ thick film. Due to the high growth rate of this process and the higher thickness of the film, the film is noticeably rougher and has numerous features visible in its STM. While the film is still rather smooth, this increased roughness means there is a substantial increase in surface area. With a larger surface area, there will be an increase in the number of surface hydrogen bonds. This will allow more electron out diffusion into adsorbates, resulting in an increased density of holes in the near surface and hence a higher sheet carrier concentration. ${ }^{18}$ The mobility of thicker films will be lower for the reasons mentioned above, and also because of the increased roughness of the surface. Further evidence for this is seen in Fig. 10, where Element $6\{100\}$ substrates generally have higher carrier concentrations and lower mobilities. This is because these are lower quality, rougher substrates and hence have a larger surface area.

Similar effects are seen with growth on polycrystalline diamond, but with the added complication of grain boundaries. Figure 7 shows it is possible to obtain STM images on this macroscopically rough material. This image shows the edge of a grain surrounded by grain boundaries. The grain area is light and reasonably uniform, as it is a single crystal within itself. However, the uniformity is not as high as in the case of the single crystal shown in Fig. 1. The grain boundary is darker as there is no surface conductivity present within this region. Therefore, when hydrogenated, polycrystalline diamond films should be more resistive in general, and this is borne out in the empirical case., ${ }^{2,18,23,24}$ Figure 8 demonstrates the reduction in sheet resistivity with substrate thickness. Initially these data are confusing, until one considers the fact that grain size increases with thickness in the case of polycrystalline diamond. This will in turn reduce the total area of grain boundaries, and thus there will be an increased area for hydrogen termination if one assumes each grain as an isolated single crystal. This is seen in Fig. 9 where the sheet carrier concentration increases with increasing thickness of substrate and hence increased grain size.

Figure 10 summarizes the above results and adds data for type $\mathrm{Ib}\{111\}$ diamond. It can be seen from this plot that there is a maximum mobility value obtainable for a particular sheet carrier concentration. This maximum is always witnessed on the Sumitomo type $\mathrm{Ib}\{100\}$ substrates. This is as expected, as $\{100\}$ orientation diamond is both the flattest and most easily polished surface, and these substrates were of higher cost and quality than the Element 6 diamonds. It is also the easiest orientation to grow on, and generally results in the flattest surface. Hence, as the flattest surface will have the least surface scattering, it stands to reason that it will yield the highest mobility in the case of surface conductivity.

$\{111\}$ diamond is seen to have a high carrier concentration and low mobility regardless of the growth conditions. Growth on $\{111\}$ diamond is technologically significantly more difficult, and usually results in rougher surfaces than growth on $\{100\}$ diamond. This could explain this result, as well as the fact that $\{111\}$ hydrogen terminated surfaces have a larger surface dangling bond density than $\{100\}$ surfaces, ${ }^{5}$ and hence there are more sites for electron out diffusion into the adsorbate layer.

These single crystals have a significantly higher maximum mobility than the polycrystalline diamonds, which are confined to the lower mobility region of the plot. It can be seen from the polycrystalline region of the plot that this material also has a maximum mobility at a specific sheet carrier concentration which is considerably lower than their singlecrystal counterparts. The mobility is presumably limited by grain boundary scattering. The data are much more scattered than the single crystal due to the variation in the substrate material. It should also be noted that one $300 \mu \mathrm{m}$ film can have a very different morphology from another and this will have substantial effects on the surface conductivity.

\section{CONCLUSIONS}

Extensive electrical characterization of the surface conductive properties of diamond grown by microwave plasma enhanced chemical vapor deposition has been demonstrated. A method for controlling a diamond film's surface electrical properties and a model explaining this peculiar behavior have been proposed. This model links the sheet carrier concentration and mobility values of a diamond film to its asso- 
ciated surface roughness, which becomes greater with the film thickness using this growth process. It has been proposed that this is due to rougher films having enhanced surface area, and hence more surface hydrogen bonds by which electrons can out-diffuse into surface based adsorbates. This leads to a higher sheet carrier concentration. The mobility is reduced by the roughness of the surface. This method allows the production of a device grade film from a low quality substrate within $10 \mathrm{~min}$. A similar process has been demonstrated on polycrystalline material, but the maximum achievable mobility is significantly lower due to grain boundary scattering. However, considerably larger area material may be realized by this technique.

\section{ACKNOWLEDGMENTS}

This work received financial support from the UK Engineering and Sciences Research Council (Grant No. GR/ R29871). One of us (O.A.W.) is also grateful to the UK Carbon Network for the funding of his postdoc position. Lakeshore Cryotronics are acknowledged for the development of Hall effect measurement apparatus and software specifically for diamond analysis.

${ }^{1}$ M. I. Landstrass and K. V. Ravi, Appl. Phys. Lett. 55, 1391 (1989).

${ }^{2}$ K. Hayashi, H. Watanabe, S. Yamanaka, H. Okushi, K. Kajimura, and T. Sekiguchi, Diamond Relat. Mater. 6, 303 (1997).

${ }^{3}$ C. Nebel, F. Ertl, C. Sauerer, M. Stutzmann, C. Graeff, P. Bergonzo, O Williams, and R. Jackman, Diamond Relat. Mater. 11, 351 (2002).

${ }^{4}$ O. Williams, M. Whitfield, R. Jackman, J. Foord, B. JE., and C. Nebel, Appl. Phys. Lett. 78, 3460 (2001)

${ }^{5}$ H. Kawarada, Surf. Sci. Rep. 26, 205 (1996).
${ }^{6}$ Y. Mori, H. Kawarada, and A. Hiraki, Appl. Phys. Lett. 58, 940 (1991). ${ }^{7}$ H. Umezawa, K. Tsugawa, S. Yamanaka, D. Takeuchi, H. Okushi, and H. Kawarada, Jpn. J. Appl. Phys., Part 2 38, L1222 (1999).

${ }^{8}$ H. J. Looi, L. Y. S. Pang, Y. Wang, M. D. Whitfield, and R. B. Jackman, Diamond Relat. Mater. 7, 565 (1998).

${ }^{9}$ A. Aleksov, A. Denisenko, U. Spitzberg, T. Jenkins, W. Ebert, and E. Kohn, Diamond Relat. Mater. 11, 382 (2002).

${ }^{10}$ R. Sung Gi, T. Mizumasa, Y. Akiba, Y. Hirose, T. Kurosu, and M. Iida, Jpn. J. Appl. Phys., Part 1 34, 5550 (1995).

${ }^{11}$ A. Denisenko, A. Aleksov, A. Pribil, P. Gluche, W. Ebert, and E. Kohn, Diamond Relat. Mater. 9, 1138 (2000).

${ }^{12}$ J. Ristein, F. Maier, M. Riedel, M. Stammer, and L. Ley, Diamond Relat. Mater. 10, 416 (2001).

${ }^{13}$ C. Sauerer, F. Ertl, C. E. Nebel, M. Stutzmann, P. Bergonzo, O. A. Williams, and R. A. Jackman, Phys. Status Solidi A 186, 241 (2001).

${ }^{14}$ A. B. Molloy, F. Jones, J. S. Foord, H. J. Looi, L. Y. S. Pang, and R. B. Jackman, Diamond Relat. Mater. 7, 550 (1998).

${ }^{15}$ O. A. Williams and R. B. Jackman, Diamond Relat. Mater. 13, 557 (2004).

${ }^{16}$ J. Ristein, M. Riedel, M. Stammler, B. F. Mantel, and L. Ley, Diamond Relat. Mater. 11, 359 (2002).

${ }^{17}$ S. G. Ri, H. Yoshida, S. Yamanaka, H. Watanabe, D. Takeuchi, and H. Okushi, J. Cryst. Growth 235, 300 (2002).

${ }^{18}$ O. A. Williams and R. B. Jackman, Semicond. Sci. Technol. 18, S34 (2003).

${ }^{19}$ C. Nebel, C. Sauerer, F. Ertl, M. Stutzmann, C. Graeff, P. Bergonzo, O. Williams, and R. Jackman, Appl. Phys. Lett. 79, 4541 (2001).

${ }^{20}$ O. A. Williams, R. B. Jackman, C. Nebel, and J. S. Foord, Semicond. Sci. Technol. 18, S77 (2003).

${ }^{21}$ B. Mantel, M. Stammler, J. Ristein, and L. Ley, Diamond Relat. Mater. 10, 429 (2001).

${ }^{22}$ R. Sung Gi, K. Tashiro, S. Tanaka, T. Fujisawa, H. Kimura, T. Kurosu, and M. Iida, Jpn. J. Appl. Phys., Part 1 38, 3492 (1999).

${ }^{23}$ O. Williams, R. Jackman, C. Nebel, and J. Foord, Diamond Relat. Mater. 11, 396 (2002).

${ }^{24}$ N. Jiang and T. Ito, J. Appl. Phys. 85, 8267 (1999). 